\title{
Postpartum Blood Loss Measurement Using Digital Image Processing
}

\author{
Siska Febrina Fauziah ${ }^{1, *}$, Suryono Suryono ${ }^{2}$, and Melyana Nurul Widyawati ${ }^{1}$ \\ ${ }^{1}$ Postgraduate Applied Midwifery, Poltekkes Kemenkes Semarang, Semarang - Indonesia \\ ${ }^{2}$ Department of Physics, Faculty of Mathematics and Natural Sciences, Diponegoro University, Semarang - Indonesia
}

\begin{abstract}
Most of maternal mortality is directly caused by obstetric hemorrhage and almost three-quarters of it take place in postpartum period. The risk of maternal death due to hemorrhage can be reduced with prompt and proper care that in turn relates to the blood loss measurement method used. Unfortunately, the most commonly practiced method is not yet accurate enough. This research proposes a postpartum blood loss measurement method using digital image processing. Initially, characterization is carried out to find out blood absorbance against digital image area and blood volume in the underpad. Results of this characterization then serve as the basis for blood loss measurement using digital image processing. This measurement basis is validated by measuring blood volume lost during delivery care. Results show that the area and volume of blood absorbed in the underpad has significant linear relation $(\mathrm{R}=0.9734)$. Digital image processing can be used to measure postpartum blood loss with a high accuracy up to $83.7 \%$. The use of this method is therefore an accurate and objective alternative to the other available method. Nonetheless, the use of this new method must also adhere to standard guidelines in delivery care as to ensure the utmost outcomes.
\end{abstract}

Keywords: Blood loss; measurement; delivery; digital image processing; accuracy.

\section{Introduction}

Accuracy in diagnose confirmation is pertinent as it determines medical interventions that in turn affects a patient's health condition. The use of health technology has therefore been an alternative in fulfilling that need. One of the technologies that has been developed for health is digital image processing. With digital image processing, the image of an object can be interpreted and hence, serves as medical clues to confirm diagnoses both accurately and objectively [1]. Computerized system that provides a framework for digital image processing can minimize errors that are usually associated to human factors. Therefore, it has the potential to improve accuracy in looking into examination results and helps medical professionals in making decision [2]. The use of computer-based systems has great advantages when compared to manual systems because it can work automatically and precisely so the system can work more efficient [3].

In obstetrics field, the accuracy in confirming a diagnosis is needed in obstetric hemorrhage cases. Most of maternal mortality is directly caused by obstetric hemorrhage and almost three-quarters of it take place in postpartum period [4]. Even though mothers do not die of bleeding, loss of blood that is not adequately treated may result in potentially fatal morbidity effects [5].
Initial treatment for hemorrhage can prevent actual bleeding and hence, prevent bleeding complications that can be life-threatening. Despite this fact, determination of blood loss is one of the major obstacles in treating hemorrhage. This difficulty relates to the measurement method being used. Accurate measurement of blood loss is very necessary in determining the proper postpartum hemorrhage diagnosis in order to prevent untimely care being given [6].

The most common method of blood loss measurement used today is visual estimation. With this method, health professionals estimate blood loss by examining the area of absorption on the underpad or the other material used in delivery. The area of blood absorption on the underpad is arbitrary that visual estimation is very subjective as it depends on the personal perception of health professionals [7]. Visual estimation method is not accurate and allows differences estimated blood volume between health professionals that may cause delay in decision making concerning the required medical intervention $[6,8]$.

Digital image processing is introduced as the new method that is capable of measuring the area of arbitrary objects accurately [9]. As the relationship between area and volume is known, it is expected to be used for measuring postpartum hemorrhage and coming up with more accurate figures. This research proposes a method

\footnotetext{
* Corresponding author: siskafebrinafauziah90@gmail.com
} 
of measuring postpartum blood loss using digital image processing based on blood absorption area on the underpad..

\section{Method}

The research is initiated by characterizing absorption on the underpad. Artificial blood is poured onto an underpad usually used for delivery. In order to obtain average area of blood on the underpad, the artificial blood is poured at a certain point gradually with varied volumes from $25 \mathrm{~mL}$ to $500 \mathrm{~mL}$, of which the accurate volume has been ensured using spuits. Each blood volume variation on the underpad is then measured for its average area, and then compared to the actual blood volume, and hence, a basis for measurement using digital image processing is set.

The basis of blood volume measurement using digital image processing is validated by measuring blood loss during delivery. Following the delivery of the baby, an underpad is placed underneath a mother's buttocks to prevent blood being mixed with amniotic fluid [10]. The blood volume absorbed on the underpad is measured once delivery care is given. The gravimetric method is used as standard reference to confirm measurement accuracy using digital image processing. Blood loss measured using gravimetric method is based on the differences in underpad weight, prior to and after delivery care use [10]. This discrepancy indicates the blood loss, in which 1 gram equals $1 \mathrm{~mL}$ [11].

Procedure for blood loss measurement in this research is shown in Fig. 1. Preparation stage is set to allow the underpad to be ready for image acquisition. Blood laded underpad is placed on a plane inside an adequately light room. Prior to image acquisition, a dummy is placed next to the underpad to be used as a spatial resolution measurement standard during image data processing. The camera used for image acquisition is of $8 \mathrm{MP}$ resolutions. Meanwhile, the angle and distance are adjusted as such as to get optimum underpad images. Distance of the camera from the underpad is 15 $\mathrm{cms}$, while the height is $100 \mathrm{cms}$. The inclination angle is $40^{\circ}$ to allow the camera to be on the wide view area. Acquired images are then transferred to the computer and are given labels to ease data processing.

Labeled images are then processed by a computer. In pre-processing stage, colored images are turned into grayscale ones, before undergoing cropping to select regions of interest (ROI). The following stage is image segmentation to differentiate images from their backgrounds. In this stage, thresholding and inversion are performed, with results of binary images, in which the object is white or given value 1, whereas the background is black, or given value 0 . This stage allows identification of object edges as to ease spatial resolution calculation.

Results of absorbed blood area calculation in this research are gained after some stages. The first stage is comparing a dummy pixel area with that of the real one, hence, 1 pixel value in $\mathrm{cm}^{2}$ is obtained. The second stage is determining absorbed blood area on the underpad by calculating the difference between the total area and the dummy area. Then, absorbed blood area in $\mathrm{cm}^{2}$ is multiplication result of absorbed blood area and 1 pixel value. Data from those stages are then processed and analyzed using a computer program to obtain blood loss in $\mathrm{mL}$. In order to ensure the accuracy of digital image processing, concordance analysis using Bland Altman framework and Mean Absolute Percentage Error (MAPE) calculation are also performed.

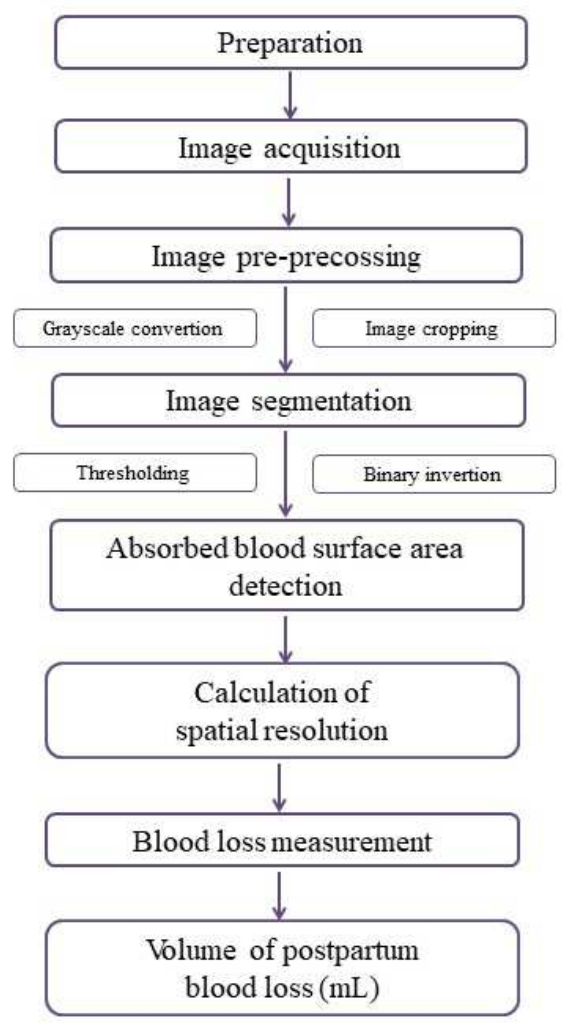

Fig. 1. Procedure of postpartum blood loss measurement using digital image processing.

\section{Result Analysis}

\subsection{Blood absorption characteristics}

Characterization of blood absorption on the underpad results in 30 blood images from 10 different sample variations. To the naked eye, patterns of blood absorption on the underpad tend to be linear, as shown in Fig. 2. The more blood poured onto an underpad, the wider the surface area is. When only a small amount of blood is poured, the result is overestimation. This is because an underpad has higher blood affinity in all direction, which allows blood be speedily absorbed and spread below and to the sides of the underpad. Once a certain volume is obtained, sidewise affinity is less, and blood is absorbed slower and hence, settles from the bottom first. Therefore, estimation results tend to be underestimation. At certain volumes when the underside of an underpad is already full, sidewise affinity is more, and hence, absorption area is wider. These two patterns 
keep on repeating themselves.

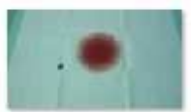

U1-25

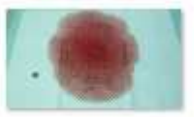

U1-150

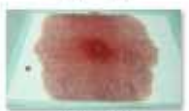

U1-275

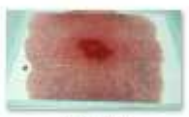

U $1-400$

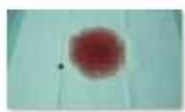

U1-50

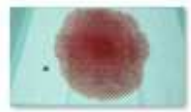

U1-175

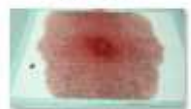

U1-300

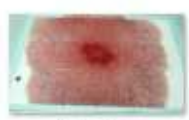

U1-425

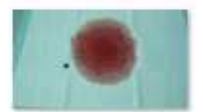

U1-75

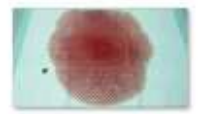

U1-200

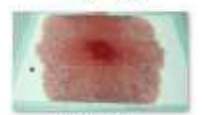

U1-325

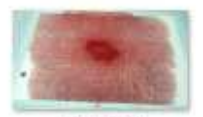

U1-450

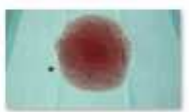

U1-100

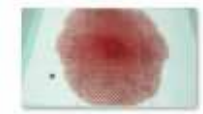

U1-225

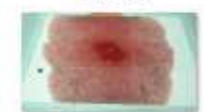

U1-350

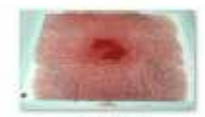

U1-475

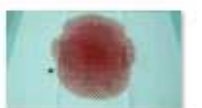

U1-125

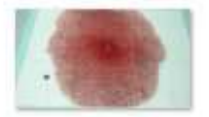

U1-250

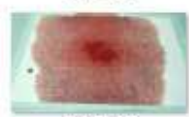

U1-375

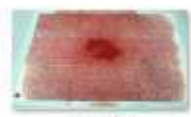

U1-500

Fig. 2. Blood absorption pattern on an underpad.

\subsection{Calculation of digital image spatial resolution}

A dummy is used as measurement standard for spatial resolution calculation in this research. Based on comparison of dummy pixel area and the actual area, it is obtained 1 pixel value in $\mathrm{cm}^{2}$. Then, calculation of spatial resolution based on that value results in average surface blood area from each blood volume variation. Comparisons of average surface blood area and actual blood volume are then put into a graph as seen in Fig. 3.
This in turn, results in an equation describing the linear relationship between blood volume and its surface area $(\mathrm{R}=0.9734)$. This is the equation that serves as the basis of blood loss measurement using digital image processing.

The used of a dummy during image acquisition also allows image acquisition to be made using all types of camera. Therefore, the use of a dummy is the one that makes digital image processing in this research better, in terms of accuracy and efficiency.

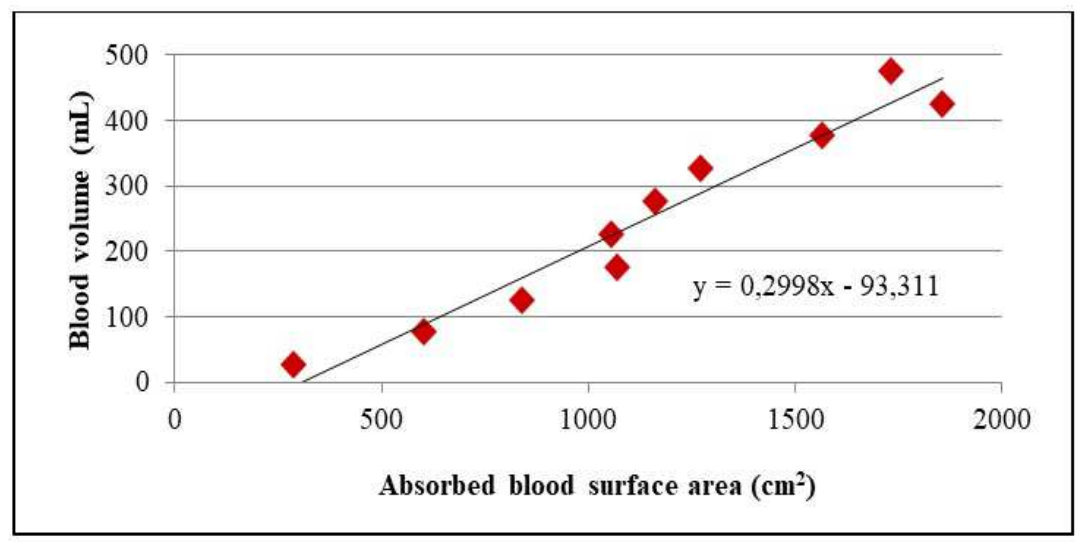

Fig. 3. Relationship of blood area and volume on an underpad.

\subsection{Blood volume measurement using digital image processing}

The process of postpartum blood loss measurement using digital image processing is shown in Fig. 4. Measurement basis from comparison of average blood area and actual blood volume is tested by carrying the same experiment as the previous stage, but with more varied volumes. Based on bland altman analysis, results of measurement using digital image processing are not significantly different with actual blood volumes as can be seen in Fig. 5., in which the bias line approaches 0, the limits of agreement is relatively narrow, and the data spread tends to be dense. Results of Mean Absolute Percentage Error (MAPE) calculation show that digital image processing has an error of $14.3 \%$, with mean difference of $30.01 \mathrm{~mL}$, compared to actual blood volumes.

Therefore, results of this research show that digital image processing can be used to measure blood loss based on blood absorption area on the underpad. The average measurement error is also relatively small that this method really has the potential to provide both accurate and objective postpartum blood loss measurement results. Determination of blood volume using digital image processing relies on the blood area visible on the underpad. It is also known that 
measurement of blood loss using digital image processing provides optimum results at certain area ranges. When the blood area is less than $312 \mathrm{~cm}^{2}$, measurement results are undefined. On the other hand, when the blood area is more than $3650 \mathrm{~cm}^{2}$, the error more than $100 \%$.

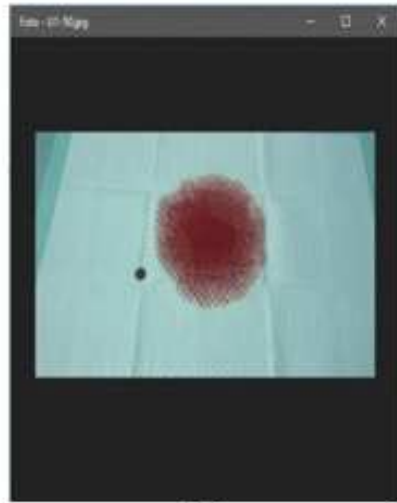

(a)

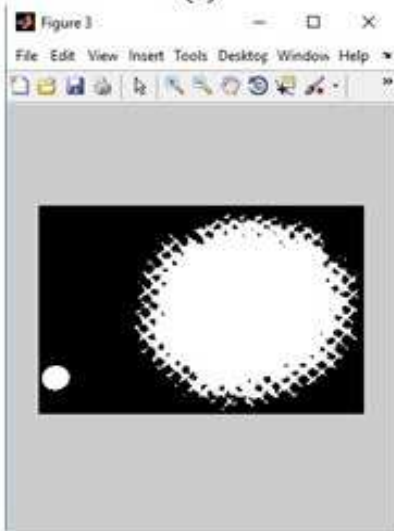

(d)

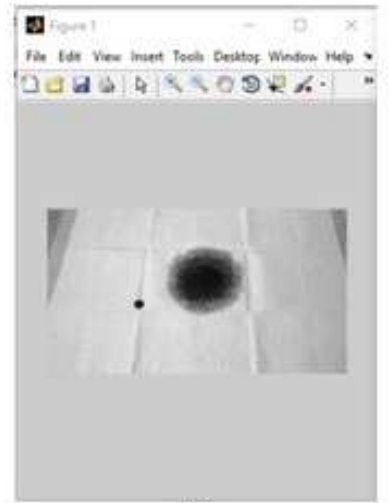

(b)

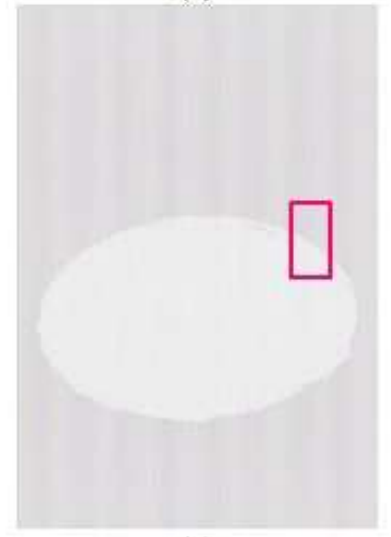

(e)

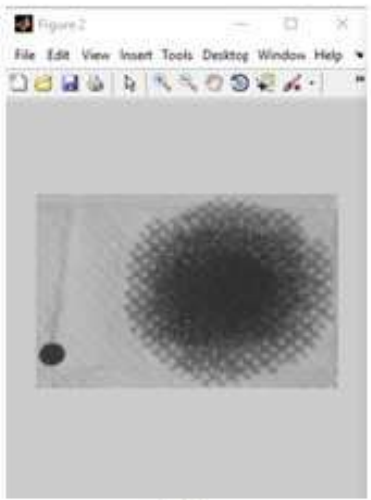

(c)

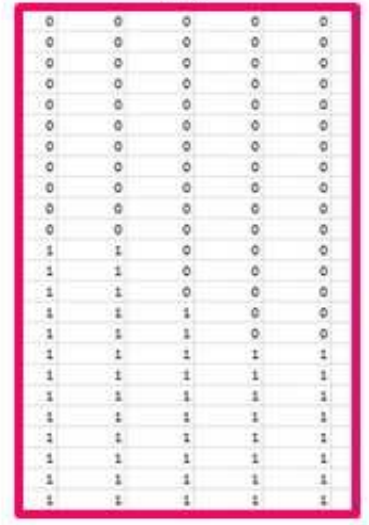

(f)

Fig. 4. Process of postpartum blood loss measurement using digital image processing; (a) RGB image, (b) grayscale image, (c) cropped image, (d) result from thresholding and inversion, (e) data image in numbers for ease of spatial resolution calculation, (f) enlarged image view.

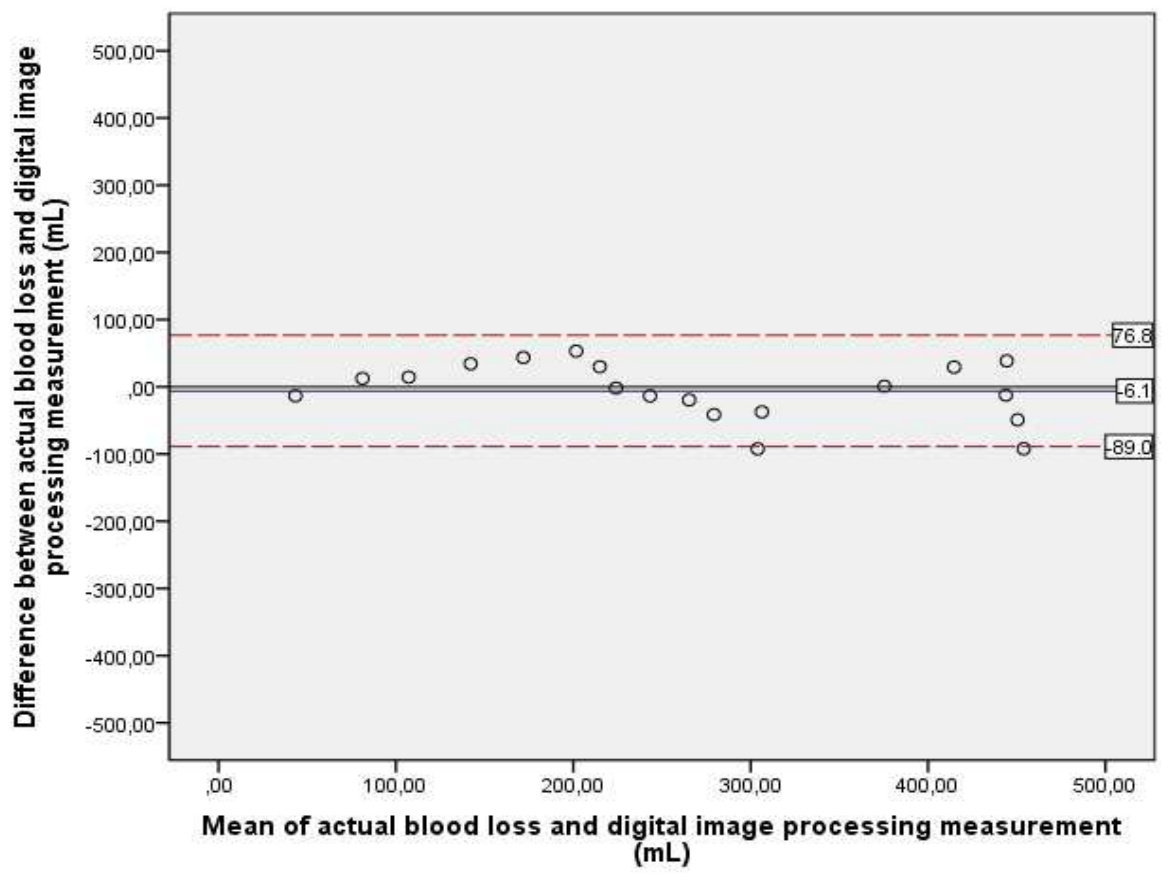

Fig. 5. Bland Altman plot describing the agreement between results from digital image processing method and actual blood volume. 
For those reasons, blood loss measurement using digital image processing is then validated by measuring blood loss during delivery care. It was then known that using standard methods, 1 out of 10 mothers experienced blood loss of more than $500 \mathrm{~mL}$. And digital image processing is capable of accurately diagnosing this type of hemorrhage case. Based on bland altman analysis, results of measurement using digital image processing are not significantly different with standard methods, as shown in Fig. 6. Furthermore, results of MAPE calculation also predict that this method is capable of postpartum blood loss measurement of $83.7 \%$ accuracy, with mean difference of $42.86 \mathrm{~mL}$, compared to the gravimetric method.

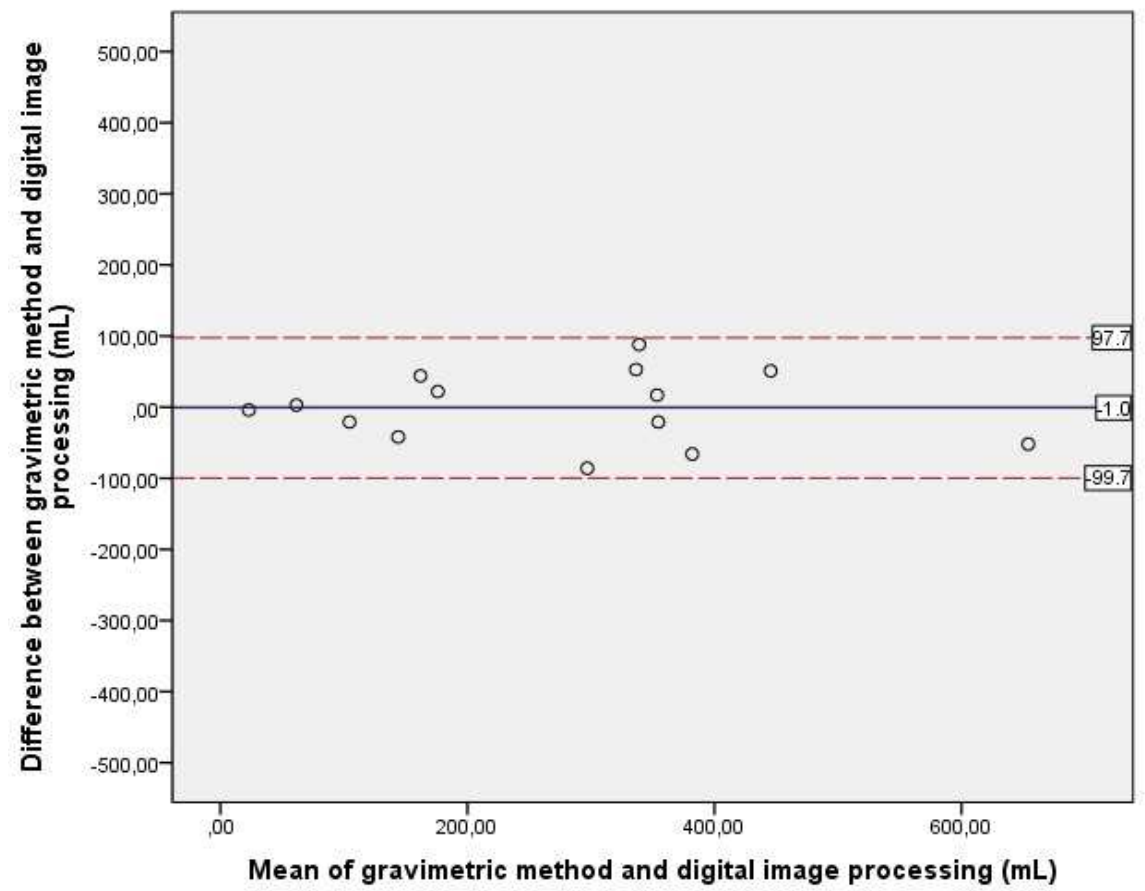

Fig. 6. Bland Altman plot describing the agreement between results of digital image processing method and results of the gravimetric method.

During implementation in delivery care, the digital image processing method has an error of only $16.3 \%$. Difference in blood loss measurement of less than $20 \%$ is still tolerable without posing any danger to the patient's condition [12]. Hence, digital image processing can be used to measure postpartum blood loss accurately, when underpads are used as the blood absorbent. Results prove that blood loss measurement can be based on blood surface area.

Digital image processing method also shows better measurement results compared to earlier research when the underpads are specially designed to be consisted of a few squares each capable of absorbing $50 \mathrm{~mL}$ of blood. Blood volume is then calculated by totaling the amount from the number of fully saturated square and half fully saturated square. Measurement of blood volume using these specially designed underpads is much better compared to the usual visual estimation method with mean difference of $80.91 \mathrm{~mL}$, compared to the gravimetric method. However, this type underpad is not widely available yet. Moreover, determination of which square is half fully saturated is not easy and may cause different perceptions as basically, the shape of the blood surface area on the underpad is arbitrary [8]. Digital image processing is capable of measuring the area of arbitrary objects accurately [9] and hence, blood loss measurement results using this method is more accurate.
This is evident with the relatively low error level and the mean difference with the gravimetric method that is almost fifty percent lower.

Nonetheless, the research carried out here has limitations as the underpads used are the ones only available for this research. Absorption characteristics may differ for each type of underpad that in turn results in different observable blood surface area. Therefore, the basis of measurement for digital image processing in this research may not fit the other types of underpads. However, characterization of blood absorption in an underpad and measurement of blood loss using digital image processing are very simple.

\section{Conclusions}

The use of digital image processing to measure postpartum blood loss is an accurate and objective alternative to the other available methods. This new method must also be supported by standard guidelines in delivery care in order to control blood absorption area on underpads. Other than that, the types of dummy and underpad used as standards for measurement of spatial resolution are also significant aspects that influence optimum results of blood loss measurement using the method developed here. 
In the future, this finding can be integrated in a long distance monitoring system of postpartum blood loss that connects postpartum mother, midwife and other medical experts. Prevention and early treatment of postpartum hemorrhage using this system is expected to be more effective and efficient. Furthermore, the community dependence on transportation to obtain health services can be reduced that indirectly support the realization of lowcarbon development.

\section{References}

1. L. Putzu, G. Caocci, and C. Di Ruberto, Artificial Intelligence in Medicine, 62, pp. 179-191, (2014).

2. S. Arslan, E. Ozyurek, and C. Gunduz $\square$ Demir, Cytometry Part A, 85, pp. 480-490, (2014).

3. S. Suryono, J. E. Suseso, C. Mashuri, A. D. Sabila, J. A. M. Nugraha, and M. H. Primasiwi, Advanced Science Letters, 23, pp. 2398-2400, (2017).

4. L. Say, D. Chou, A. Gemmill, Ö. Tunçalp, A.-B. Moller, J. Daniels, et al., The Lancet Global Health, 2, pp. e323-e333, (2014).

5. Z. Karaca, B. A. Laway, H. S. Dokmetas, H. Atmaca, and F. Kelestimur, Nature reviews. Disease primers, 2, pp. 16092-16092, (2016).
6. G. Lilley, D. Burkett-St-Laurent, E. Precious, D. Bruynseels, A. Kaye, J. Sanders, et al., International journal of obstetric anesthesia, 24, pp. 8-14, (2015).

7. N. Golmakani, K. Khaleghinezhad, S. Dadgar, M. Hashempor, and N. Baharian, Iranian Journal of Nursing and Midwifery Research, 20, pp. 471-475, (2015).

8. L. Wilcox, C. Ramprasad, A. Gutierrez, M. Oden, R. Richards-Kortum, H. Sangi-Haghpeykar, et al., Maternal and Child Health Journal, pp. 1-8, (2016).

9. S. Suryono, K. Kusminarto, G. B. Suparta, and A. Sugiharto, International Journal of Applied Engineering Research, 10, pp. 35499-35503, (2015).

10. AWHONN, Journal of Obstetric, Gynecologic, \& Neonatal Nursing, 44, pp. 158-160, (2015).

11. D. J. Vitello, R. M. Ripper, M. R. Fettiplace, G. L. Weinberg, and J. M. Vitello, Journal of veterinary medicine, 2015, (2015).

12. M. Brooks, G. Legendre, S. Brun, P. E. Bouet, L. P. Mendes, B. Merlot, et al., Scientific Reports, 7, p. 46333, (2017). 